\title{
Interactive comment on "Characteristics of the main primary source profiles of particulate matter across China: from 1987 to 2017' by Xiaohui Bi et al.
}

\section{Anonymous Referee \#2}

Received and published: 23 September 2018

This study reviewed particle chemical composition profiles from typical emission sources measured in China, based on the 374 published profiles in literature and 2870 profiled conducted by the research team. Source profile is vital in source apportionment and pollution controls, and localized source information is important for accurate source identification and contribution estimation. The review made a significant contribution to this important area. But the manuscript in its present form needs clear clarification and revisions on its data analysis and presentation. My comments on this manuscript are as follows:

About literature search and paper selection, as done in many review studies, more de- 
tails on the literature search and selection should be provided and evaluated. For example, how many people did the search and were they done independently? Are there any duplicated papers from different searchers and different database? How many papers found in total in the first round of the search? What're the inclusion/exclusion criteria? Was there any evaluation on the quality control and assurance in the decision of inclusion/exclusion? Line 147 and figure 2, - clarification of the sampling method into these three groups is inappropriate. e.g. "medium-volume" could be used in "dilution sampling" -they considered different factors instead of different approaches of the same factor. The authors should rethink of the classification and reanalyze the part. A similar problem of classification is in the biomass burning part- Biomass boiler, FCE (field combustion experiment), LCS (laboratory chamber study), the first refers to the burning facility, while the other two are experiment methods. How could these three be in parallel here? The study analyzed sources in "coal combustion, industrial emissions, vehicle emissions, dust, cooking emissions, biomass burning" - some are based on fuel type, some are sectoral difference- this mixed classification should be corrected. e.g. "coal combustion and industrial emissions"- is coal burned in many industrial factories? What's the burning fuel in industrial combustion process? Residential burning is a significant part of coal combustion, particularly in China. But the study did not have analysis and discussion on this. This important source should not be missed. Line 166, was TOT method used in EC/OC analysis? Line 180, retene is also one widely used tracers for biomass burning, especially wood combustion. Line 263, as seen in Figure 1 there is a significant number of coal emission studies, and even higher than the number of vehicle emissions. "rarely" might be inappropriate here. Line 425- may be good to provide some local studies on biomass burning emissions from indoor stoves and open burning. Line 427 and figure 10- as mentioned above, "biomass boiler" was not a "sampling method". because of the problem in such classification, the discussion from line 430 needs to be revised. Line 428, is "biomass boiler" the boilers used in industry or household? What about the home stoves? Line 433-434- high EC or BC emissions are usually due to the domination of high temperature flaming burning conditions. Lien 
437-438, it seems that the authors only considered open burning when referring to "FCE". Lines 441-443, a recent study showed that for open biomass burning, emission factors of most air pollutants from field measurements and simulated chamber studies in laboratory are comparable. Line 446, also due to high combustion temperature and flaming dominance burning conditions. Line 527, the reference "Yan et al., 2017" is missing Line 555-556, as mentioned above the biomass boilers are burning facilities while the other two are sampling/experiment methods. Line 600, please consider enlarging and improving the resolution Giving different burning conditions and different fuel properties even for the same type of fuel, within the current compiled database, it is interesting to look into the spatial difference in source profiles for the same source type. Generally, the manuscript is understandable but could be improved after a language edit and polish. Please go through the manuscript and check for grammars and spelling errors for example, Line 79, "source measurement is actually it is time to ..." the sentence is incomplete. Please check and revise

Interactive comment on Atmos. Chem. Phys. Discuss., https://doi.org/10.5194/acp-2018-687, 2018. 